\title{
Synthesis of Linear Antenna Arrays
}

\author{
P. K. MURTHY AND A. KUMAR, MEMBER, IEEE
}

Abstract-Synthesis of antenna arrays employing the $L_{2}$-norm as well as the $L_{c o}$-norm is discussed. The approximation in the $L_{\infty}$-norm is obtained making use of Lawson's algorithm. A general iterative perturbation technique has been evolved for pattern synthesisfor the case when the antenna currents alone are vaned as well as for the case when both the antenna currents and the element positions are simultaneously varied. A few illustrative examples are given. The convergence of the iteration and the uniqueness of the solution are discussed.

\section{INTRODUCTION}

A considerable amount of work [1], [2] has been done on the synthesis of linear equally-spaced antenna arrays. These methods are based on Fourier series expansion, minimization of maximum deviation, and interpolation theory. Shaped beam radiation patterns have been synthesized by Stutzman [3] using the iterative sampling method.

Spacing the elements unequally along a straight line in order to obtain a better performance is a more recent innovation in array synthesis theory. Probably, the first work on unequally spaced arrays has been carried out by Unz who has given several methods [4]-[10] for pattern synthesis. Sahalos [11] further

Manuscript received September 1, 1975; revised April 11, 1976.

P. K. Murthy was with the Department of Electrical Communication Engineering, Indian Institute of Science, Bangalore, India. He is now with the Rockets and Missiles Laboratory, Department of Aeronautical Engineering, Indian Institute of Science, Bangalore, India

A. Kumar is with the Department of Electrical Communication Engineering, Indian Institute of Science, Bangalore, India. 
extended Unz's orthogonal method to synthesize three-dimensional arrays. Ma [12] has used mini-max criterion and has applied techniques of approximation theory while Ishimaru [13] has described a theory of unequally spaced arrays based on the Poisson's summation formula. Recently Mautz and Harrington [14] have described a method wherein pattern synthesis is carried out with a constraint on the source norm.

The problem of choosing the element positions instead of specifying them has been tackled by Perini and Idselis [15] by making use of the steepest descent technique. Mikuni and Nagai [16] have used a modified steepest descent technique to obtain a set of antenna currents and element positions which results in restricting the synthesized pattern to lie within a specified region. Redlich [17] has made use of a perturbation technique to arrive at a set of element positions and the corresponding currents which minimize the mean squared error between the desired and synthesized patterns. However, no information is available regarding the convergence of the iteration and uniqueness of the solution.

In this paper, a method to synthesize antenna arrays employing either the $L_{2^{-}}$or the $L_{\infty}$-norm is discussed. The approximation in the $L_{\infty}$-norm is obtained by making use of Lawson's algorithm. Described in this paper is an iterative perturbation technique to synthesize an arbitrary pattern by varying both the element positions and the antenna currents. Uniqueness of the solution has been studied numerically and it has been found that the sets of antenna currents and element positions that yield the least error may not always be unique. A particular case of this problem, namely, when only antenna currents are allowed to vary, is also discussed.

\section{Theory of PatternSynthesis}

Let it be desired to design an $N$-element array whose array factor $f_{\mathrm{s}}(\vec{I}, \bar{X} ; \phi)$ approximates an arbitrarily prescribed pattern $f_{d}(\phi)$ best in a given norm. This amounts to the determination of the set of currents $\bar{I}$ and the set of element positions $\bar{X}$ such that the error

$$
\sigma=\left\|f_{d}-f_{s}\right\|
$$

is minimum.

This may be solved by an iterative perturbation technique described below. Consider a center-symmetric $N(=2 n)$-element linear array (Fig. 1) whose array factor after $(l-1)$ iterations have been carried out, is given by

$$
f_{S I}^{l}(\bar{I}, \bar{X} ; \phi)=\sum_{k=1}^{n} I_{k}^{l} u_{k}^{l}\left(x_{k}^{l} ; \phi\right)
$$

where

$$
u_{k}\left(x_{k}^{l} ; \phi\right)=\cos \psi_{k}^{l}, \quad \psi_{k}^{l}=\beta x_{k}^{l} \cos \phi+\alpha
$$

and $\alpha_{k}^{l}=-\beta x_{k}{ }^{l} \cos \phi_{0}$ with $\phi_{0}=$ steering angle and $\beta=2 \pi / \lambda$. Adopting the $L_{p}$-norm, the error $\sigma_{p}^{l}$ after (I - 1) iterations is given by

$$
\sigma_{p}^{l}=\left\|f_{d}-f_{S I}{ }^{l}\right\|_{p}=\left[\int_{a}^{b}\left|f_{d}-f_{S I}^{l}\right|^{p} w d \phi\right]
$$

where $w(\phi)$ is a nonnegative weight function. The set of currents is now determined such that $\sigma_{p}{ }^{l}$ is minimum. Having obtained the currents, the element positions are perturbed to obtain a better match, i.e.,

$$
x_{k}^{l} \rightarrow x_{k}^{l}+\delta_{k}^{l}, \quad k=1,2, \cdots, n .
$$

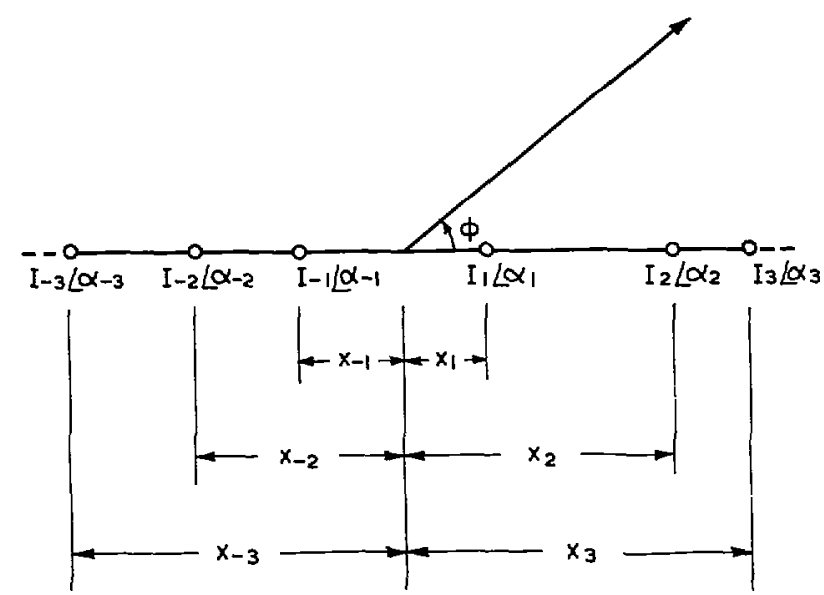

Fig. 1. Center-symmetric linear array.

Correspondingly the $u_{k}{ }^{l}$ change as given below:

$$
u_{k}^{l} \rightarrow \cos \psi_{k}^{l} \cos \left(\beta \delta_{k}^{l} \cos \phi\right)-\sin \psi_{k}^{l} \sin \left(\beta \delta_{k}^{l} \cos \phi\right) .
$$

Assuming the $\delta_{k}{ }^{l}$ to be small, to a first-order of approximation,

$$
\cos \left(\beta \delta_{k}^{l} \cos \phi\right)=1 \text { and } \sin \left(\beta \delta_{k}^{l} \cos \phi\right)=\beta \delta_{k}^{l} \cos \phi .
$$

Therefore,

$$
u_{k}^{l} \rightarrow u_{k}^{l}-\beta \delta_{k}^{l} \cos \phi \sin \psi_{k}^{l} .
$$

The new array factor $f_{S X}{ }^{l}$ corresponding to this perturbation is given by

$$
f_{S X}^{l}=f_{S I}^{l}-\sum_{k=1}^{n} \delta_{k}^{l} V_{k}^{l}\left(x_{k}^{l} ; \phi\right)
$$

where

$$
V_{k}^{\prime}=I_{k}^{2} \beta \cos \phi \sin \psi_{k}^{l}
$$

The error $\xi_{p}{ }^{\mathrm{I}}$ is now defined as

$$
\begin{aligned}
\xi_{p}^{l} & =\left\|f_{S X^{l}}{ }^{\prime}-f_{d}\right\|_{p}=\left\|\left(f_{S I}^{l}-f_{d}\right)-\sum_{k=1}^{n} \delta_{k}^{l} V_{k}^{l}\right\|_{p} \\
& =\left\|e^{l}-\sum_{k=1}^{n} \delta_{k}^{l} V_{k}^{l}\right\|_{p}, \quad \text { where } e^{l}=\left(f_{S I}{ }^{l}-f_{d}\right) .
\end{aligned}
$$

The $\delta_{k}{ }^{l}$ are now determined so that $\xi_{p}{ }^{l}$ is minimum. Minimization of $\xi_{p}{ }^{l}$ may be seen to be similar to the minimization of $\sigma_{p}{ }^{l}$. The procedure for determining the $\delta_{k}^{l}$ consists in approximating the error function e' by $\sum_{k=1}^{n} \delta_{k}^{l} V_{k}^{l}$. Since e' itself is small, the $\delta_{k}{ }^{l}$ may be expected to be small, thusjustifying the approximation made in (6). It is expected that with $\delta_{k}{ }^{l}$ so chosen, a closer approximation becomes possible, i.e.,

$$
\xi_{p}^{l}<\sigma_{p}^{l} .
$$

The iteration process may be continued until no further reduction in the error becomes possible. However, the iterative process described above does not converge for the case $p=\infty$, if there exists a point $\phi_{m}$ where $e^{l}$ is an extremum and simultaneously $\left.\sum_{k=1}^{n} \delta_{k}^{l} V_{k}^{l}\right|_{\phi=\phi_{m}}=0$. This may be proved as follows.

Since $e^{l}$ is the difference between $f_{d}$ and its best mini-max approximation $f_{S I}^{l}[18]$,

$$
\left\|e^{l}\right\|_{\infty}=\left|e^{l}\left(\phi_{m}\right)\right| .
$$


Further, since

$$
\begin{gathered}
\sum_{k=1}^{n} \delta_{k}^{l} V_{k}^{l}\left(\phi_{m}\right)=0, \\
\xi_{\infty}^{l}=\left\|e^{l}-\sum_{k=1}^{n} \delta_{k}^{l} V_{k}^{l}\right\|_{\infty} \geq\left\|e^{l}\right\|_{\infty}=\sigma_{p}^{l} .
\end{gathered}
$$

Hence. the iteration does not converge.

It may be noted that this condition occurs in the synthesis of the Gaussian pattern, exp $\left[-|a|\left(\phi-\phi_{0}\right)^{2}\right]$ at $\phi=\phi_{0}$. In such cases the method described above may be modified as follows.

i) $f_{d}$ is normalized so that $f_{d}\left(\phi_{0}\right)=1$.

ii) The set of currents are obtained by minimizing $\left|f_{1} f_{S I}{ }^{\prime}-f_{d}\right|^{\prime}$.

iii) The error $\xi_{\infty}{ }^{l}$ is redefined as

$$
\xi_{\infty}^{l}=\left\|\left\{f_{S I^{l}}-\left(\sum_{k=1}^{n} I_{k}\right) f_{d}\right\}-\sum_{k=1}^{n} \delta_{k}^{l} V_{k}^{l}\right\|_{\infty} .
$$

Noting that $\left[f_{S_{I}}-\left(\sum_{k=1}^{n} I_{k}\right) f_{d}\right]_{\phi=\phi_{0}}=0$, the $\delta_{k}^{l}$ may now be obtained by minimizing $\xi_{x} l$.

Note that the expressions in (3), (10) and (14) are all of the form $\left|f f-\sum_{k=1}^{n} a_{k} g_{k}\right|_{: p}^{\prime}$. Minimization of this expression is equivalent to obtaining the best approximation in the $L_{p}$-norm of the function $\boldsymbol{f}$ by $\sum_{k=1}^{n} a_{k} g_{k}$, where the $g_{k}$ are given. Choice of $\boldsymbol{p}=2$ leads to the least squares approximation and $p=\infty$ leads to the mini-max approximation. Both of these cases are considered here.

Case No. $1: p=2$

Given that the $g_{k}$ are linearly independent, this problem may be solved as given below.

i) A new set of orthonormal functions $\stackrel{*}{\mathscr{g}}_{k}$ are obtained from the $g_{k}$ such that

$$
\stackrel{*}{g}_{i}=\sum_{j=1}^{i} b_{i j} g_{j}, \quad i=1,2, \cdots, n
$$

and $\left(\stackrel{*}{g}_{i} \stackrel{*}{g}_{j}\right)=\delta_{i j}$ where the inner product of two functions $\boldsymbol{f}$ and $\mathrm{g}$ is given by

$$
(f, g)=\int_{a}^{b} f(\phi) g(\phi) w(\phi) d \phi
$$

$w(\phi)$ being a given nonnegative weight function. The $b_{i j}$ are obtained by making use of the Gram-Schmidt procedure [19].

ii) The least squares approximation to $f$ is now given by [19],

$$
\sum_{k=1}^{n}\left(f, \stackrel{*}{g}_{k}\right) \stackrel{*}{g}_{k}
$$

Identifying the $g_{k}$ with $u_{k}$, an explicit expression for $I_{k}{ }^{l}$ may now be obtained from the following identity.

Let $C_{k}=\left(f_{d}, u_{k}\right)$, then

$$
\begin{aligned}
f_{S I}^{l}= & \sum_{k=1}^{n} I_{k}^{l} u_{k} \equiv \sum_{k=1}^{n}\left(f_{d}, u_{k}\right) \sum_{j=1}^{k} b_{k j} u_{j} \\
= & C_{1}\left(b_{11} u_{1}\right)+C_{2}\left(b_{21} u_{1}+b_{22} u_{2}\right)+\cdots \\
& +C_{k}\left(b_{k 1} u_{1}+b_{k 2} u_{2}+\cdots+b_{k k} u_{k}\right)+\cdots \\
& +C_{n}\left(b_{n 1} u_{1}+b_{n 2} u_{2}+\cdots+b_{n n} u_{n}\right) \\
= & \left(C_{1} b_{11}+C_{2} b_{21}+\cdots+C_{k} b_{k 1}+\cdots\right. \\
& \left.+C_{n} b_{n 1}\right) u_{1}+\cdots \\
& +\left(C_{k} b_{k k}+C_{k+1} b_{(k+1), k}+\cdots\right. \\
& \left.+C_{n} b_{n k}\right) u_{k}+\cdots+\left(C_{n} b_{n n}\right) u_{n} .
\end{aligned}
$$

Hence

$$
I_{k}^{l}=\sum_{j=k}^{n}\left(f_{d},{ }^{*} u_{j}\right) b_{j k}
$$

Similarly, $\delta_{k}^{l}$ may also be determined.

Case No. $2: p=\infty$

Mini-max approximation over a finite point set only is considered. Let $f_{d}$ be specified over a finite point set @. Then,

$$
\|\left. f_{d}\right|_{\infty}=\sup _{\phi_{i} \in \Phi}\left|f_{d}\left(\phi_{i}\right)\right| \text {. }
$$

It has been proved elsewhere [18], [20] that the best minimax approximation may be obtained by computing a certain weighted least squares approximation. This method is advantageous because there are several areas (for instance, vectorvalued functions and functions of complex variable) where there arc no known algorithms for $L_{x}$-approximation but where least squares approximation can be used. Lawson's algorithm consists in generating the required weight function.

Lawson's algorithm for La,-approximation: A sequence of weight functions $w^{k}\left(\phi_{i}\right)=w_{i}^{k}$ with $\sum_{\phi_{i} \in \Phi} w_{i}^{k}=1$ is defined below

$$
w_{i}^{k+1}=\frac{w_{i}^{k}\left|f_{i}-\sum_{k=1}^{n} a_{k} g_{k i}\right|}{\sum_{\phi i \in \Phi} w_{i}^{k}\left|f_{i}-\sum_{k=1}^{n} a_{k} g_{k i}\right|}
$$

Lawson [20] has shown that this sequence of weights does converge and the corresponding least squares approximation is also the best mini-max approximation, i.e., defining

$$
\sigma_{2}{ }^{k}=\left\|f-\sum_{k=1}^{n} a_{k} g_{k}\right\|_{2},
$$

the numbers $\sigma_{2}{ }^{k}$ increase monotonically and converge to $\stackrel{*}{\sigma}$ defined by

$$
\stackrel{*}{\sigma}=\max _{\phi_{i} \in \Phi}\left|f_{i}-\sum_{k=1}^{n} a_{k} g_{k i}\right|=\lim _{k \rightarrow \infty} \sigma_{2}{ }^{k} .
$$

The convergence is slow without an acceleration scheme. It has been observed that Lawson's algorithm tends to drive the weights to zero everywhere except at the extremal points of the error curve. Hence attempts may be made to speed up the algorithm by making the weights tend to zero as rapidly as possible everywhere except at the extremal points. The scheme due to Rice and Usow [21] is described here.

Step 1 : "l" iterations of Lawson's algorithm are carried out (a typical value of $l$ is $\mathbf{3}$ or $\mathbf{4}$ ).

Step 2: $w_{i}^{k}$ is set to zero, if

$$
\left|f_{i}-\sum_{k=1}^{n} a_{k} g_{k i}\right| \leq \frac{\left[\sigma_{2}{ }^{k}\right]^{2}}{\max _{\phi_{i} \in \Phi}\left|f_{i}-\sum_{k=1}^{n} a_{k} g_{k i}\right|} .
$$

Step 3: Iteration is started from step 1 again.

\section{SYNTHESIS BY AMPLITUDE CONTROL}

Using the techniques described in the preceding section the given pattern is synthesized by varying the magnitude of antenna currents alone. In order to illustrate the method a smoothly varying Gaussian pattern is synthesized. Further, to qualitatively assess the effect of the nature of the desired pattern on the quality of the approximation obtained, a sector-beam pattern 


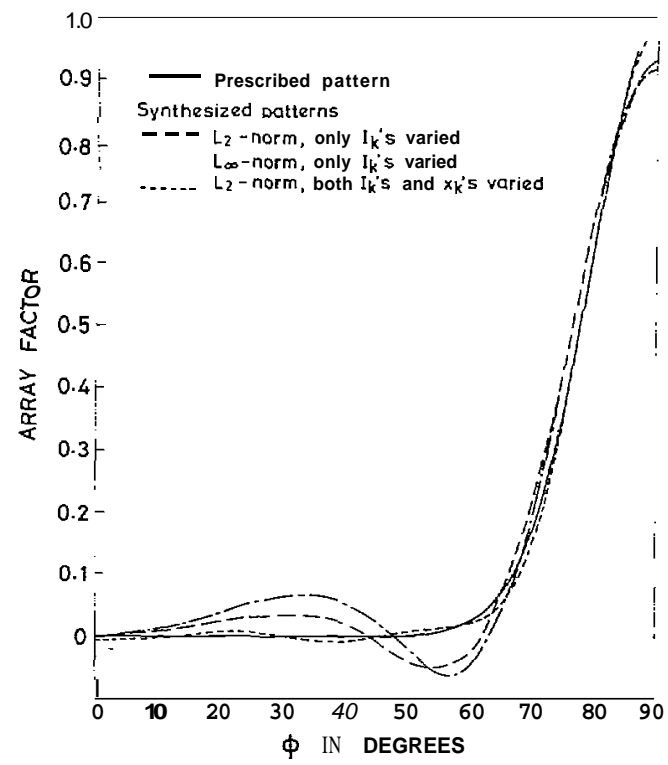

Fig. 2. Synthesis of Gaussian pattern.

with a finite discontinuity is also synthesized. For the sake of computational simplicity a 6-element center-symmetric array with $\lambda / 2$ element spacing is chosen. Both patterns are taken to be symmetric about $\phi=\pi / 2$. So that $\alpha=0$.

\section{Illustration I =Synthesis of Gaussian Pattern}

The pattern to be synthesized is defined below:

$$
f_{d}=\exp \left[-15\left(\phi-\frac{\pi}{2}\right)^{2}\right], \quad 0 \leq \phi \leq \pi
$$

Case 1: $p=2$

Since the pattern is symmetric about $\phi=\pi / 2$, it is sufficient to consider the pattern only in the range $(0,(\pi / 2))$. The weight function is chosen to be a constant and in light of the normalization condition,

$$
\int_{0}^{\pi / 2} w(\phi) d \phi=1, \quad w(\phi)=\frac{2}{\pi} .
$$

The currents obtained using (18) are

$$
I_{1}=0.43224, \quad I_{2}=0.32067, \quad I_{3}=0.15787 .
$$

The mean squared error $\sigma_{2}=1.61338 \times 10^{-3}$. The desired and synthesized patterns are shown in Fig. 2.

The error $\sigma_{2}$ depends upon the desired pattern, the element spacing, and the number of elements. It is of interest, therefore, to determine the minimum number of elements and the corresponding spacing so that the error does not exceed a prescribed limit. For this case, the error has been studied [2] numerically as a function of both $\mathrm{N}$ and $d / \lambda$.

Note that Schelkunoff's method would aim at choosing the antenna currents such that the error

$$
\sigma_{2}=\int_{-\pi}^{\pi}\left|f_{d}(\phi)-f_{s}(\phi)\right|^{2} d \psi
$$

with $\psi=\pi \cos \phi$, is minimum. This is equivalent to the minimization of

$$
\int_{0}^{\pi}\left|f_{d}(\phi)-f_{s}(\phi)\right|^{2} \sin \phi d \phi
$$

whereas in the method described above, the error defined by

$$
\sigma_{2}=\int_{0}^{\pi}\left|f_{d}-f_{s}\right|^{2} w(\phi) d \phi
$$

is minimized. Thus, on comparison, it may be found that Schelkunoff's method is a particular case of the method presented here with $\sin \phi$ chosen as the weight function.

Case 2: $p=\infty$

The Gaussian pattern described by (23) is specified over 181 equally spaced points, i.e.,

$$
\begin{aligned}
f_{d}\left(\phi_{i}\right) & =f_{d i}=\exp \left[-15\left(\phi_{i}-\pi / 2\right)^{2}\right], \quad i=1,2, \cdots, 181 \\
\phi_{i} & =(i-1) \frac{71}{2 \times 180^{\prime}}
\end{aligned}
$$

Accelerated Lawson's algorithm is started choosing the initial weights to be equal, i.e.,

$$
w_{i}=\frac{\ldots}{\ldots}, \quad i=1,2, \cdots, 181 .
$$

The iteration has been found to converge to a third decimal accuracy in 8 iterations. The weight function and the synthesized pattern are shown in Fig. 2. The currents are obtained to be

$$
I,=0.43308, \quad I_{2}=0.31621, \quad I_{3}=0.18415 .
$$

The maximum deviation may be seen to be only 0.067 as compared to 0.0892 for the case $p=2$.

\section{Illustration 2: Synthesis of Sector-Beam Pattern}

The sector-beam pattern is defined below:

$$
f_{d}= \begin{cases}0, & 0 \leq \phi<\pi / 3 \\ 1, & \pi / 3 \leq \phi \leq 2 \pi / 3 \\ 0, & 2 \pi / 3<\phi \leq \pi\end{cases}
$$

As in the previous example, $w(\phi)=2 / \pi$. The currents for this case are found to be

$$
I_{1}=0.90449, \quad I_{2}=0.28928, \quad I_{3}=-0.15324
$$

and the mean squared error is $\sigma_{2}=2.61981 \times 10^{-2}$. The desired and synthesized patterns are shown in Fig. 3.

\section{Synthesis by Simultaneous Control of Antenna CURRENTS AND ELEMENT POSITIONS}

Employing the method described in Section II, the Gaussian pattern and the sector-beam pattern are synthesized by varying both the magnitudes of the antenna currents and the element positions. A numerical study of the uniqueness of the solution has been carried out by starting the iteration from different sets of initial element positions. It appears that the solution is not always unique and depends upon the pattern to be synthesized.

\section{Illustration I : Synthesis of Gaussian Pattern}

The Gaussian pattern given by (23) is synthesized employing $L_{2}$-norm by a 6-element, center-symmetric broadside array. The weight function, as before, has been chosen to be a constant equal to $2 \pi 1$. Five sets of initial element positions are chosen and in all these cases the iterative process has converged to the same solution. However, the number of iterations required to obtain the solution depends upon the initial choice of element positions. The synthesized pattern is shown in Fig. 2. The initial element 
TABLE I

Synthesis of Gaussian Pattern

\begin{tabular}{|c|c|c|c|c|c|c|c|c|c|}
\hline \multirow[b]{2}{*}{ Number } & \multicolumn{3}{|c|}{$\begin{array}{l}\text { Initial Element Positions } \\
\text { in Wavelengths }\end{array}$} & \multicolumn{3}{|c|}{$\begin{array}{l}\text { Final Element Positions } \\
\text { in Wavelengths }\end{array}$} & \multicolumn{3}{|c|}{ Antenna Currents } \\
\hline & $x_{1}^{\prime}$ & $x_{2}^{\prime}$ & $x_{3}^{\prime}$ & $x_{1}$ & $x_{2}$ & $x_{3}$ & $I_{1}$ & $I_{2}$ & $I_{3}$ \\
\hline & 0.25 & 0.75 & $\begin{array}{l}1.45 \\
1.5\end{array}$ & \multirow{2}{*}{0.344} & \multirow{2}{*}{1.038} & \multirow{2}{*}{1.752} & \multirow{2}{*}{0.577} & \multirow{2}{*}{0.322} & \multirow{2}{*}{0.090} \\
\hline & $\begin{array}{l}0.35 \\
0.35\end{array}$ & $\begin{array}{l}1.05 \\
0.85\end{array}$ & $\begin{array}{l}1.75 \\
1.35\end{array}$ & & & & & & \\
\hline
\end{tabular}

TABLE II

Synthesis of Sector-Beam Pattern

\begin{tabular}{|c|c|c|c|c|c|c|c|c|}
\hline \multirow[b]{2}{*}{ Number } & \multicolumn{3}{|c|}{$\begin{array}{l}\text { Final Element Positions } \\
\text { in Wavelengths }\end{array}$} & \multicolumn{3}{|c|}{ Final Antenna Currents } & \multirow[b]{2}{*}{$100 \sigma_{2}$} & \multirow{2}{*}{$\begin{array}{l}\text { Number of } \\
\text { Iterations }\end{array}$} \\
\hline & $x_{1}$ & $x_{2}$ & $x_{3}$ & $I_{1}$ & $I_{2}$ & $I_{3}$ & & \\
\hline 1 & $\begin{array}{c}(0.25) \\
0.2488\end{array}$ & $\begin{array}{c}(0.75) \\
0.5879\end{array}$ & $\begin{array}{l}(1.25) \\
1.5496\end{array}$ & 0.8376 & 0.2916 & -0.2282 & 1.7727 & 14 \\
\hline 2 & $\begin{array}{l}(0.30) \\
0.2950\end{array}$ & $\begin{array}{c}(0.90) \\
0.7786\end{array}$ & $\begin{array}{l}(1.25) \\
' 1.5353\end{array}$ & 1.0091 & 0.3295 & -0.2350 & 1.7730 & 179 \\
\hline 3 & $\begin{array}{r}(0.325) \\
0.3048\end{array}$ & $\begin{array}{r}(0.975) \\
0.8839\end{array}$ & $\begin{array}{r}(1.625) \\
1.5204\end{array}$ & 1.0406 & 0.1070 & -0.2424 & 1.7735 & 132 \\
\hline 4 & $(0.225)$ & $(0.475)$ & $(0.925)$ & The iter & tion doe & not conve & & \\
\hline
\end{tabular}

Note: Initial element positions are given in brackets.

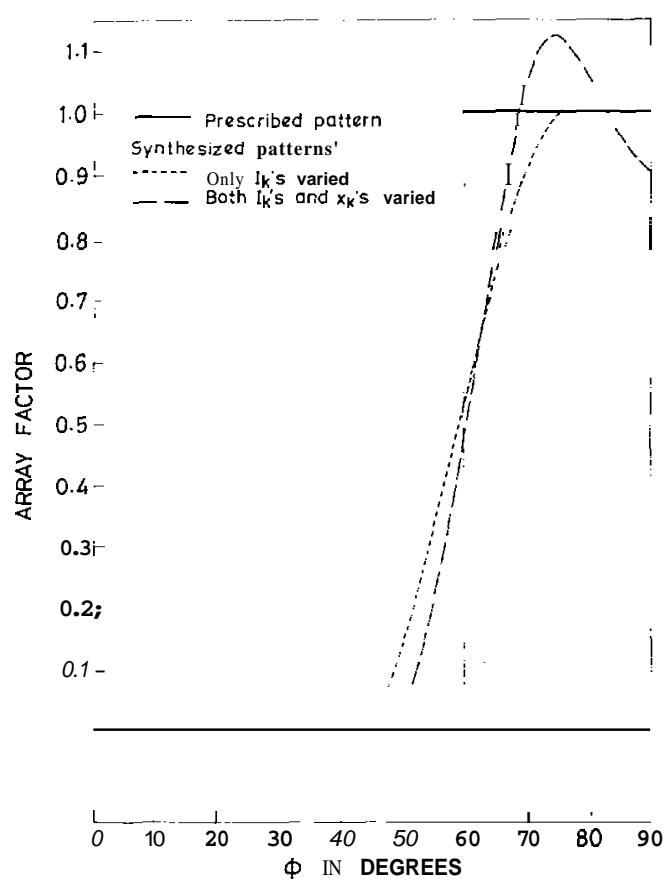

Fig. 3. Synthesis of sector-beam pattern using $L_{2}$-norm

positions, the final element positions and the antenna currents are presented in Table J.

\section{Illustration 2: Synthesis of Sector-Beam Pattern}

The sector-beam pattern as defined by (27) is synthesized employing $L_{2}$-norm as before, by a 6-element, center-symmetric broadside array. The weight function is chosen to be a constant equal to $2: \pi$. The iteration has been started from four different sets of initial element positions. The results of the synthesis are shown in Table II.
Note that each choice of initial element position has led to a different set of final element positions and antenna currents. However, it has been found that the pattern of any of these arrays is the same, as well as the mean-squared error. Thus it appears that the optimum pattern is unique but the element positions and antenna currents for a given pattern are not unique. The desired and synthesized patterns are shown in Fig. 3.

\section{Discussion}

Use of Lawson's algorithm in pattern synthesis reduces the problem of mini-max approximation to one of weighted least squares approximation. Thus mini-max approximation may be obtained even when the functions $u_{i}$ are complex. This implies that arrays of arbitrary geometry may be designed using the techniques presented here.

When linear arrays with element positions prescribed are synthesized using the $L_{\infty}$ - or $L_{2}$-norm, the current distribution obtained is unique in light of a uniqueness theorem [18]. In the case of $L_{x}$-approximation, however, it may be noted that the $u_{i}$ must constitute a Chebyshev set for the solution to exist. This condition imposes a constraint on the length of the array for a given $N$ as pointed out by Ma [12]. The closeness of the match between the desired and the synthesized pattern appears to depend upon the nature of the desired pattern (see Figs. 2 and 3). The match is better for smooth pattern and for a pattern with a finite discontinuity Gibb's phenomenon is observed.

In the case of synthesis when both the element positions and antenna currents are allowed to vary and when the iteration is started from different initial element positions, different sets of element positions and antenna currents are arrived at depending upon rhe prescribed pattern. Thus, in the case of the synthesis of sector-beam pattern each choice of initial element positions has led to a different solution; each solution corresponding to a local minimum. However, the synthesized pattern and hence $\sigma_{2}$ are the same for all the solutions though $\bar{X}$ and $\bar{I}$ are different. It appears that the synthesized pattern is unique though 
the $\bar{X}$ and $\bar{I}$ that yield this pattern are not. In general, any choice of initial element positions leads to a local minimum only. To obtain a global minimum the iteration must be initiated from several choices of initial element positions and the resulting local minima must be compared to obtain the global minimum. A convenient choice of initial element positions is the one where the interelement spacings are equal. A digital computer may readily be programmed to accomplish this scheme.

\section{ACKNOWLEDGMENT}

The authors are thankful to Prof. B. S. Ramakrishna and also to Prof. N. S. Nagaraja for the facilities provided. They are also grateful to Prof. H. M. Barlow, FRS, for his valuable criticism of this work.

\section{REFERENCES}

[1] R. E. Collin and F. J. Zucker, Antenna Theory-Part 1. New York: McGraw-Hill, 1969, pp. 138-305.

[2] P. K. Murthy, "Synthesis of antenna arrays," Ph.D. Thesis, Indian Institute of Science, Bangalore, 1975.

[3] W. 1. Stutzman, "Synthesis of shaped-beam radiation patterns using the iterative sampling method," IEEE Trans. Antennas Propagat. vol. AP-19, pp. 36-41, Jan. 1971

[4] H. Unz, "Antenna arrays with arbitrarily distributed elements," Doctoral dissertation, Electrical Engineering Department, Univ. of California, Berkeley, Calif., Nov. 1956.

[5] "Linear arrays with arbitrarily distributed elements," Electron. Res. Lab., Univ. of California, Berkeley, Calif. Ser. 60, Issue 48, pp. 1-58, Nov. 1956.

[6] - "Linear arrays with orbitrarily distributed elements." IEEE Trans. Antennas Propagat., vol. AP-8, pp. 222-223, March 1960.

[7] "Matrix relations for a linear array with dipole elements in the Fresnel zone," IEEE Trans. Antennas Propagat., vol. AP-9, p. 220, March 1961.

[8] __ "Non-uniform arrays with spacings larger than one wavelength," $\overrightarrow{I E E E}$ Trans. Antennas and Propagat., vol. AP-10, pp. 647-648, Sept. 1962.

[9] "Non-uniformly spaced arrays: The orthogonal method," Proc. IEEE, vol. 54, pp. 53-54, Jan. 1966.

[10] "Non-uniformly spaced arrays: The Eigenvalues method," Proc. IEEE, vol. 54, pp. 676-678, April 1966.

[11] J. Sahalos, "A solution of the general non-uniformly spaced antenna array," Proc. IEEE, vol. 62, pp. "1292-1294, Sept. 1974.

[12] M. T. Ma and L. C. Walters, "Synthesis of non-uniformly spaced antenna arrays yielding approximately equal sidelobes," Radio Sri. I (New Series), vol. 1, pp. 601-608, May 1966.

[13] A. Ishimaru, "Theory of unequally-spaced arrays," IEEE Trans. Antennas Propagar., vol. AP-10, pp. 691-702, Nov. 1962.

[14] J. R. Mautz and R. F. Harrington, "Computational methods for antenna pattern synthesis," IEEE Trans. Antennas Propagar., vol AP-23, pp. 507-512, July 1975.

[15] J. Perini and M. Idselis. "Note on antenna pattern synthesis using numerical iterative methods," IEEE Trans. Antennas Propagat., vol. 19, pp. 284-286, March 1971.

[16] Y. Mikuni and K. Nagai, "On a method of optimization in pattern synthesis of array antennas," 1972 IEEE G-AP Symposium Digest, Williamsburg, Virginia.

[17] R. W. Redlich, "Iterative least squares synthesis of non-uniformly" spaced linear arrays," IEEE Trans. Antennas Propagar., vol. AP-21, pp. 106-108, Jan. 1973 .

[18] J. R. Rice, The Approximation of functions, Vol. I. New York: Addison-Wesley, 1956.

[19] P. 1. Davis, Interpolation and Approxitnarion. New York: Blaisdell, 1963.

[20] C. L. Lawson, "Contributions to the theory of linear least maximum approximations," Ph. D. Thesis, UCLA, 1961.

[21] J. R. Rice and K. H. Usow, "Lawson's algorithm and extensions," Math. Com, vol. 22, pp. 118-127, Jan. 1968.

\section{A Generalized Network Formulation for Aperture Problems}

\section{ROGER F. HARRINGTON, FELLOW, IEEE, AND} JOSEPH R. MAUTZ, SENIOR MEMBER, IEEE

\begin{abstract}
A general formulation for aperture problems is given in terms of the method of moments. It applies to any two regions isolated except for coupling through the aperture. The aperture characteristics
\end{abstract}

Manuscript received November 29, 1975; revised March 21, 1976 This work was sponsored by Air Force Cambridge Research Laboratories under Contract F19628-73-C-0047 and by the National Science Foundation under Grant GK-38309.

The authors are with the Department of Electrical and Compute Engineering, Syracuse University, Syracuse, NY 13210.

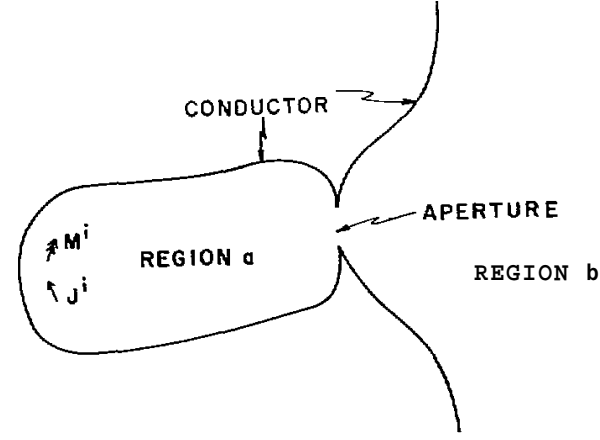

Fig. 1. General problem of two regions coupled by aperture.

are expressed in term of two aperture admittance matrices, one for each region. The admittance matrix for one region is independent of the other region, and hence can be used for any problem involving that region and aperture. The solution can be represented by two generalized n-port networks connected in parallel with current sources. The current sources are related to the tangential magnetic field which exists over the aperture region when the aperture is closed by an electric conductor. Formulas for fields (linear functionals) and power (quadratic functionals) are given in terms of the admittance matrices.

\section{INTRODUCTION}

The general problem of coupling through apertures has many specific applications, such as apertures in a conducting screen, waveguide-fed apertures, cavity-fed apertures, waveguide-towaveguide coupling, waveguide-to-cavity coupling, and cavityto-cavity coupling. The literature on these problems is extensive. Many books, of which [1]-[4] are typical, discuss the problem and give references to some of the literature.

This paper formulates the problem in terms of a moment solution of the operator equation. An application of the equivalence principle separates the problem into two parts, namely, the regions on each side of the aperture. The only coupling is through the aperture, whose characteristics can be expressed by aperture admittance matrices, one for each region. These admittance matrices depend only on the region being considered, being independent of the other region. The aperture coupling is then expressible as the sum of the two independent aperture admittance matrices, with source terms related to the incident magnetic field. This result can be interpreted in terms of generalized networks as two n-port networks connected in parallel with current sources. The resultant solution is equivalent to an $n$-term variational solution.

Since the problem is divided into two mutually exclusive parts, one can separately solve a few canonical problems, such as apertures in conducting screens, in waveguides, and in cavities, and then combine them in the various permutations mentioned above. Computer programs can be developed for treating broad classes of canonical problems, such as apertures of arbitrary shape in conducting planes, in square waveguides, and in rectangular cavities. Such programs can then serve as broad and versatile tools for designing electromagnetic networks with aperture coupling.

\section{General Formulation}

Fig. 1 represents the general problem of aperture coupling between two regions, called region $a$ and region $b$. In region $a$ there are impressed sources $\boldsymbol{J}^{i}, \boldsymbol{M}^{i}$, and region $b$ is assumed source free. The more general case of sources in both region $a$ and region $b$ can be treated as the superposition of two problems, one with sources in region a only, plus one with sources in 